\title{
ORIGINAL
}

\section{A CORRELATIONAL STUDY BETWEEN FINE NEEDLE ASPIRATION CYTOLOGY AND HISTOPATHOLOGY OF PALPABLE BREAST LUMPS}

\author{
Vilas P. Kulkarni ${ }^{1}$, Abhijit S. Rayate ${ }^{1}$, Cristine Ann Thomas ${ }^{1}$, Nikhil R. Barhate ${ }^{1}$, Gaurav J. \\ Gaikwad $^{1}$, Basawraj S. Warad ${ }^{1}$, Basawraj S. Nagoba ${ }^{2}$ \\ ${ }^{1}$ Department of Surgery, Maharashtra Institute of Medical Sciences \& Research, Latur, India \\ ${ }^{2}$ Department of Microbiology, Maharashtra Institute of Medical Sciences \& Research, Latur, India
}

Corresponding author: Abhijit S. Rayate

E-mail: asrayate@yahoo.com

\begin{abstract}
Incidence of breast malignancies has been rising. Any breast lump needs to differentiated as surgery may not be required in all cases. The National Health Services Breast Screening Program recommends Fine Needle aspiration cytology (FNAC) as a non-operative diagnostic tool. FNAC is a cheap, readily available, relatively painless, repeatable and patient-friendly investigation with good sensitivity and specificity. FNAC does not yield a tissue diagnosis as opposed to core biopsy, however, a well-performed FNAC and reporting by an expert pathologist help to avoid unnecessary surgeries in benign lesions where only conservative management or elective surgery is needed. Aims: To study the correlation between FNAC and histopathological reports of palpable breast lumps. Materials and method: We conducted an observational study at a tertiary care hospital. The study included 237 patients with palpable breast lumps who had undergone FNAC, excisional biopsy and histopathology in the same institution. The results of FNAC and histopathology reports were correlated into benign and malignant categories. Results: There were 225 women and 12 men in this study. FNAC had reported 20 malignant $(C 4+C 5)$ cases and 217 benign cases. Histopathology revealed 21 malignant cases and 216 benign cases. We observed a sensitivity of $90.48 \%$, specificity of $99.54 \%$, a positive predictive value of 95\%, a negative predictive value of $99.08 \%$, a concordance of $92.41 \%$, a diagnostic accuracy of $98.73 \%$ and a negative likelihood ratio of 0.1 for FNAC. Conclusions: Besides other advantages, FNAC of a breast lesion is reliable enough to rule out malignancy.
\end{abstract}

Keywords: palpable breast lump, Fine needle aspiration cytology, histopathology, benign, malignant

\section{Introduction}

Breast cancer is the leading cancer in women. Hyderabad district has ranked first in breast cancer $(48.0$ per 100,000$)$ among all populationbased cancer registry (PBCRs) [1]. The incidence rate increased significantly by a range of $2.9 \%$ to $8.2 \%$ annually across different cancer registries during 1988-2016. The age-specific cancer incidence rate for breast cancer in females has increased with increase in age and has peaked in the age group 50-69 years. Israel (84.6 per 100,000) and Lille in France (115.4 per 100,000) had the highest incidence of breast cancer in Asia and the world respectively [1].

Triple assessment of breast lesion is practiced worldwide for definitive diagnosis. It comprises of clinical examination, imaging and cyto- 
histological diagnosis [2], [3]. Previously, the pathological diagnosis involved invasive methods like incisional/excisional biopsy under anesthesia which usually was an inpatient procedure. It was invariably associated with some delay in histopathology reports, repeated admission for definitive surgery, mental, social, and physical inconvenience [4].

Fine Needle Aspiration Cytology (FNAC) gives a definitive nonoperative diagnosis which if malignant, can hasten further management. A definitive diagnosis of benign etiology helps to plan an appropriate conservative treatment or elective surgery if needed [3]. Though core biopsy is considered statistically more valuable and more informative [3], [5], FNAC may be the only available modality in developing countries, especially in rural centers or peripheral referral units.

Ever since the pioneering work at the Karolinska Institute, FNAC has been a simple, safe, easily available, cheap, patient friendly, minimally invasive procedure without need for anesthesia [6], [7]. Also, FNAC does not require a patient to with-hold any anticoagulant drugs [7].

Different studies have shown wide variability in the sensitivity of FNAC ranging from $35 \%$ to $95 \%$ and the specificity ranging from $48 \%$ to $100 \%$ [5], [7]-[9]. False-negative reports on FNAC are still possible at a significant rate due to diagnostic errors and true-false negative factors. Errors in diagnosis may occur due to low expertise, case-overload and inadequate correlation with clinico-radiological findings. Improper technique, small or mobile lesions, islands of atypia can also lead to false-negative results. In instances of inconclusive or indeterminate reports, USG guided FNAC improves the effectiveness of the test [10].

FNAC has proved itself to be a competent tool for nonoperative diagnosis of breast lumps, especially in developing countries [8]. Surgical excision will be required in cases where cytology reports and clinico-radiological findings don't co-relate. [7].

A well-performed FNAC and reporting by an expert pathologist help to avoid above pitfalls. Hence, we have conducted this study with an objective to co-relate FNAC and histology findings in a breast.

\section{Materials and Methods}

We have conducted a descriptive observational study based on a review of records of patients of palpable breast lumps at Maharashtra Institute of Medical Sciences \& Research (Medical College), Latur, India, from December 2016 to Jan 2020. We have included only those patients who had undergone both FNAC and histopathology at our institution to maintain the same set of pathologists and to minimize bias. We found 237 such patients and accessed and analyzed their FNAC and final histopathology reports. We had categorized the cytology reports according to National Health Services Breast Screening Program (NHSBSP) [3], [11]. Even though only reports were accessed, we had taken patient consent and maintained patient confidentiality. The Institutional Ethics committee had approved the study. We had analyzed the data using Microsoft Excel and SPSS.

We did not come across any $\mathrm{C} 1$ records for the obvious reason that a surgeon will not plan a definitive surgery based only on a $\mathrm{C} 1$ report. A $\mathrm{C} 1$ finding always mandates a repeat-FNAC with sonographic guidance if required or a core biopsy in clinically suspicious cases. For analytical purposes, we have considered $\mathrm{C} 2$ and $\mathrm{C} 3$ as benign category and $\mathrm{C} 4$ and $\mathrm{C} 5$ as a malignant category.

We had defined the statistical parameters for our study. We defined sensitivity as the ability of FNAC to detect malignancy (true-positive) in patients who did have breast malignancy. Specificity was defined as the probability of being FNAC-negative (benign lesion) when there were no malignant changes on histopathology. Accuracy is the proportion of the correct results (true-positive and true-negative) to all the cases studied. Positive Predictive Value (PPV) is the probability of having breast malignancy following a positive FNAC finding. Negative Predictive Value (NPV) is the probability of not having breast malignancy following negative FNAC findings.

\section{Results}


We observed a maximum incidence of cases in the age group 31 to 45 years $(45.99 \%)$ followed by the age group 14 to 30 years (35.59\%). The mean age was 34.05. There were 225 women and 12 men in this study. Table no. 1 shows the category-wise distribution of FNAC findings of these patients.

\begin{tabular}{lcc}
\hline Categories & Description & $\begin{array}{c}\text { Number of } \\
\text { cases }\end{array}$ \\
\hline C2 & Benign & 205 \\
\hline C3 & $\begin{array}{c}\text { Atypical } \\
\text { probably } \\
\text { benign }\end{array}$ & 12 \\
\hline C4 & $\begin{array}{c}\text { Suspicious of } \\
\text { malignancy }\end{array}$ & 7 \\
\hline C5 & Malignancy & 13 \\
\hline & Total & 237 \\
\hline
\end{tabular}

\section{Table 1 - Category wise FNAC findings}

Table 2 shows the details of FNAC findings and their subsequent correlation with histopathology. FNAC had reported 20 cases as malignant $(\mathrm{C} 4+\mathrm{C} 5)$ and 217 cases as benign. Histopathology revealed 21 malignant cases and 216 benign cases. The common findings on FNAC were fibroadenoma (126 patients; $53.16 \%)$ and fibrocystic changes (21.52\%). There were 13 FNAC reports for malignancy and seven reports of suspicious for malignancy. On histopathology, there were 125 cases of fibroadenoma and 56 cases of fibrocystic disease. Histopathology confirmed all the cases of gynecomastia. The cases of mastitis included cases of granulomatous mastitis, acute mastitis, lactational mastitis, and antibioma.

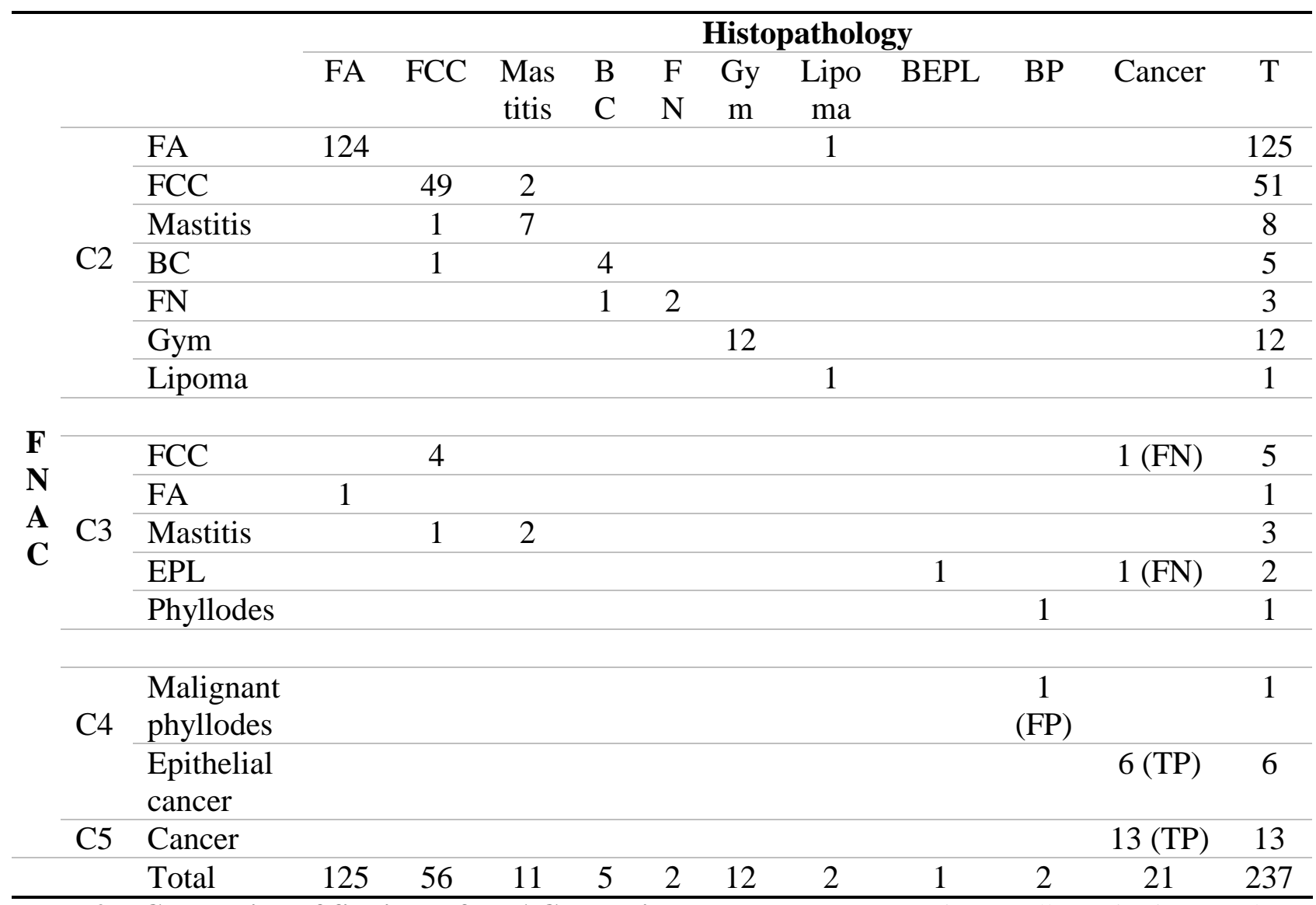

Table 2 - Correlation of findings of FNAC and histopathology (FNAC: Fine needle aspiration cytology, FA: Fibroadenoma, FCC: Fibrocystic changes, BC: Benign cyst, FN: Fat necrosis. Gym: Gynecomastia, EPL: Epithelial proliferative lesion, BEPL: Benign epithelial proliferative lesion, BP: Benign Phyllodes TP: True positive, FN: False negative, FP: False positive)

Invasive ductal carcinoma was the diagnosis in 20 malignant cases. Six cases of C4 and all C5 cases were invasive carcinoma (19 true-positive
$\{\mathrm{TP}\}$ cases). One case of fibrocystic changes with mild atypia on FNAC turned out to be invasive ductal carcinoma on histopathology. 
One case of the epithelial proliferative lesion with atypia which was reported as $\mathrm{C} 3$ on FNAC, turned out to be ductal carcinoma in situ. Thus, our study had two false-negative $(\mathrm{FN})$ cases. FNAC had reported a case as phyllodes tumor with suspicion for malignancy but on histopathology, it was benign phyllodes (falsepositive: $\mathrm{FP}=1)$. So, there were 215 truenegative cases (TN). Table 3 shows the statistical representation between FNAC and histopathology.

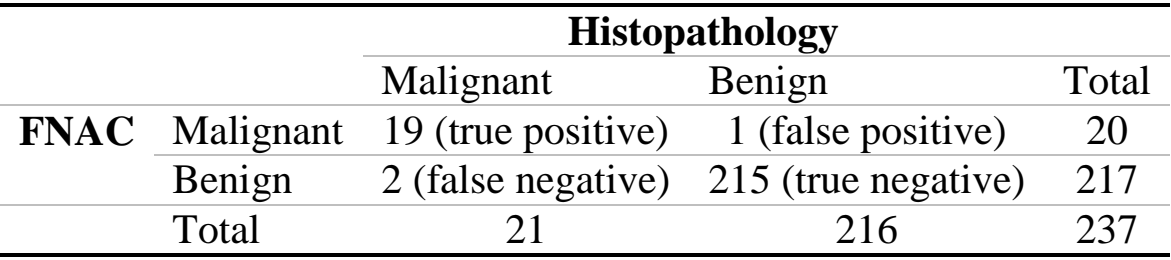

Table 3 - Statistical comparison between FNAC and histopathology

The statistical calculations revealed the following values:

Sensitivity: TP/ $(\mathrm{TP}+\mathrm{FN}) \mathrm{X} 100=19 /(19+2)$ $\mathrm{X} 100=90.48 \%(95 \%$ CI $69.62 \%$ to $98.83 \%)$

Specificity: TN/ $(\mathrm{TN}+\mathrm{FP}) \mathrm{X} 100=215 /$ $(215+1) \mathrm{X} 100=99.54 \%(95 \%$ CI $97.45 \%$ to 99.99\%)

PPV: TP/ $(\mathrm{TP}+\mathrm{FP}) \mathrm{X} 100=19 /(19+1) \mathrm{X} 100$ $=95.00 \%$ (95\% CI $72.79 \%$ to $99.26 \%)$

NNV: TN/ $(\mathrm{TN}+\mathrm{FN}) \mathrm{X} 100=215 /(215+2)$ $\mathrm{X} 100=99.08 \%$ (95\% CI $96.64 \%$ to $99.75 \%)$

Positive Likelihood Ratio $=195.43(95 \%$ CI 27.52 to 1387.93 )

Negative Likelihood Ratio $=0.10(95 \% \mathrm{CI}$ 0.03 to 0.36 )

Accuracy $(\mathrm{TP}+\mathrm{TN}) /(\mathrm{TP}+\mathrm{TN}+\mathrm{FP}+\mathrm{FN}) \mathrm{X} 100$ $=98.73 \%$ (95\% CI $96.35 \%$ to $99.74 \%$ )

Concordance: consistent agreement between FNAC and histopathology $=219 / 237=92.41 \%$.

\section{Discussions}

We had observed a mean age of 34.05 years (age range 14 to 80 years). About $46 \%$ of the patients were between 31 to 45 years of age and $35.59 \%$ between 14 to 30 years of age. In a similar study by Hebbar et al., the age range was 16 to 74 years (mean age 41.68 years) [12]. Khemka et al. showed similar age patterns and age group with maximum patients was 40-44 years [4]. Pudasaini et al. had observed a mean age of 35.9 years in their study [13]. In a study by Mitra et al; the age range was 15 to 69 years with $36.8 \%$ of the patients between 30 to 40 years of age [14]. But, Panjvani et al. had observed maximum incidence in the age group 21-30 [15]. Panwar et al. had $60 \%$ study participants below 30 years of age [16].

In our study, the most common benign breast disease on histology was fibroadenoma $(53.16 \%)$. This finding was comparable with previous studies like Panwar et al. (53.7\%), Sankaye et al. (46.56\%) and Kujur et al. (43.39\%) [16]-[18]. In our study, maximum cases of malignancy belonged to age group 46 to 60. Saadat et al had observed 43.6 years as the mean age and most of the patients in the 36-45 years age group [19].

Statistical results from our study were comparable with other studies as shown in Table 4 [12]-[17], [20]-[23].

In our study, FNAC was able to predict the same diagnosis as on histopathology in 219 cases hence the concordance was $92.41 \%$. In the study by Panwar et al., the concordance in C2, C3, and C4 lesions was 97\% [16]. Overall diagnostic accuracy was 98.73 and comparable with previous studies [15], [21], [23]. Negative likelihood ratio in our study was 0.1 which means that if the FNAC result was negative, the probability that this patient has breast carcinoma is approximately $10 \%$. Yu et al. had observed a negative likelihood ratio of 0.08 [8]. We observed a false-negative rate of $9.5 \%$ which was comparable with previous studies [12], [13]. 


\begin{tabular}{lccccc}
\hline \multicolumn{1}{c}{ Study } & $\begin{array}{c}\mathbf{n}=\text { cases of } \\
\text { histopathology }\end{array}$ & $\begin{array}{c}\text { Sensitivity } \\
(\boldsymbol{\%})\end{array}$ & $\begin{array}{c}\text { Specificity } \\
(\boldsymbol{\%})\end{array}$ & $\begin{array}{c}\text { PPV } \\
(\boldsymbol{\%})\end{array}$ & $\begin{array}{c}\text { NPV } \\
(\boldsymbol{\%})\end{array}$ \\
\hline Hebbar et al. [12] & 96 & 93.1 & 100 & 100 & 90.47 \\
\hline Pudasaini et al. [13] & 73 & 93 & 100 & - & - \\
\hline Mitra et al. [14] & 68 & 84.6 & 72.4 & - & - \\
\hline Panjvani et al. [15] & 90 & 97.82 & 100 & 100 & 97.82 \\
\hline Panwar et al. [16] & 108 & 100 & 97 & - & - \\
\hline Sankaye et al. [17] & 76 & 88.37 & 96.42 & 97.43 & 84.37 \\
\hline Aker et al. [20] & 733 & 98.02 & 90.58 & 96.48 & 94.54 \\
\hline Miskovic et al. [21] & 100 & 97.7 & 89.1 & 95.5 & 94.2 \\
\hline Takhellambam et al. [22] & 61 & 90.48 & 100 & 100 & 95.23 \\
\hline Alema et al. [23] & 75 & 83.3 & 100 & 100 & 98.6 \\
\hline Our study & 237 & 90.47 & 99.54 & 95 & 99.08 \\
\hline
\end{tabular}

Table 4 - Comparison of our study with other studies (PPV: Positive predictive value, NPV: negative predictive value)

NHSBSP recommend a repeat aspiration for C4 reports with low cellularity. In cases with good cellularity but suspicious of malignancy, core biopsy (image-guided, if required) should be preferred over repeat FNAC. A C3 or C4 cytology report should not dictate the need of a definitive surgery [3]. Clinical assessment and imaging findings should support the diagnosis before operative intervention. This might be the reason why many studies compare only $\mathrm{C} 2$ and C5 reports for statistical purposes.

Core biopsy tends to misread DCIS and invasive carcinoma which may coexist with atypical ductal hyperplasia. Such lesions will need surgical excision [7]. In many cases, FNAC and even core biopsy can confuse between fibroadenoma and benign phyllodes [7].

We observed some limitations to our study. Our study sample may not adequately represent the general population. Non-palpable lesions and USG guided FNAC for the same were not included. The study aimed at correlation between reports of FNAC and histopathology, so we were not able to focus a lot on other characteristics of the lesion and operative details.

\section{Conclusion}

Our study was able to establish a good positive correlation between the FNAC and histopathology reports, hence we recommend FNAC as a reliable tool for a non-operative pathological diagnosis during the triple assessment. We agree with Miskovic et al. that
FNAC allows a minimally invasive, rapid diagnosis but should be corelated with clinical and imaging findings, thus reducing the risk of missed diagnosis of breast cancer [21].

It should be borne in mind that a negative result neither solves patient's concerns nor rules out the possibility of malignancy. If clinical suspicion is strong, a core biopsy or a surgical intervention may be warranted for a definitive histopathological diagnosis. FNAC does not preserve the histological architecture, which limits its ability to make a definitive diagnosis. However, rapid diagnosis by FNAC can shorten or avoid hospital admission and speed a patient's route to an appropriate specialist. It also reduces the pressure on financial resources necessary for surgical procedures for the confirmation of the diagnosis especially in developing countries like India.

A well-performed targeted FNAC, an expert pathologist and a multidisciplinary correlation help to avoid unnecessary surgeries in nonneoplastic and benign nodules of breast where only conservative management or followup is needed.

Acknowledgement: The Authors express their gratitude to Dr. N.P. Jamadar (Dean) for permission and support during the study.

\section{References}

[1] "Report of National Cancer Registry Programme (2012-2016).” ICMR- National 
Centre for Disease Informatics and Research, Bengaluru, India, pp. 1-282, 2020, [Online]. Available:

https://www.ncdirindia.org/All_Reports/Report _2020/resources/NCRP_2020_2012_16.pdf.

[2] R. C. Sainsbury, "The Breast," in Bailey \& Love's Short Practice of Surgery, 27th ed., N. Williams, P. R. O'Connell, and A. McCaskie, Eds. Boca Raton: CRC press, 2018, p. 863.

[3] A. H. Lee et al., Guidelines for non-operative diagnostic procedures and reporting in breast cancer screening, vol. G 150. London: The Royal College of Pathologists, 2016.

[4] A. Khemka, N. Chakrabarti, S. Shah, and V. Patel, "Palpable Breast Lumps: Fine-Needle Aspiration Cytology versus Histopathology: a Correlation of Diagnostic Accuracy," Internet J. Surg., vol. 18, no. 1, pp. 1-13, 2008.

[5] S. M. Willems, C. H. M. van Deurzen, and P. J. van Diest, "Diagnosis of breast lesions: fineneedle aspiration cytology or core needle biopsy? A review.," J. Clin. Pathol., vol. 65, no. 4, pp. 287-92, Apr. 2012.

[6] J. A. Linsk, "Aspiration cytology in sweden: The karolinska group," Diagn. Cytopathol., vol. 1, no. 4, pp. 332-335, Oct. 1985.

[7] E. Łukasiewicz, A. Ziemiecka, W. Jakubowski, J. Vojinovic, M. Bogucevska, and K. Dobruch-Sobczak, "Fine-needle versus coreneedle biopsy - which one to choose in preoperative assessment of focal lesions in the breasts? Literature review," J. Ultrason., vol. 17, pp. 267-274, Dec. 2017.

[8] Y. H. Yu, W. Wei, and J. L. Liu, "Diagnostic value of fine-needle aspiration biopsy for breast mass: A systematic review and meta-analysis," BMC Cancer, vol. 12, no. 1, p. 41, 2012.

[9] M. Wang, X. He, Y. Chang, G. Sun, and L. Thabane, "A sensitivity and specificity comparison of fine needle aspiration cytology and core needle biopsy in evaluation of suspicious breast lesions: A systematic review and meta-analysis.," Breast, vol. 31, pp. 157166, Feb. 2017.

[10] J. J. Patel, P. C. Gartell, P. B. Guyer, A. Herbert, and I. Taylor, "Use of ultrasound localization to improve results of fine needle aspiration cytology of breast masses," J. R. Soc. Med., vol. 81, no. 1, pp. 10-12, Jan. 1988.

[11] I. O. Ellis, S. Humphreys, M. Michell, S. E. Pinder, C. A. Wells, and H. D. Zakhour, "Guidelines for non-operative diagnostic procedures and reporting in breast cancer screening. NHSBSP publication no. 50." NHS Cancer Screening Programmes, Sheffield, pp. 160, 2001, [Online]. Available: www.cancerscreening.nhs.uk.

[12] A. Hebbar and H. Iyanna, "Prospective study of fine needle aspiration cytology of clinically palpable breast lump with histopathological correlation," Int. J. Res. Med. Sci., vol. 1, no. 3, pp. 257-262, 2013.

[13] S. Pudasaini and O. P. Talwar, "Study of fine needle aspiration cytology of breast lumps and its histopathological correlation in Pokhara Valley.," Nepal Med. Coll. J., vol. 13, no. 3, pp. 208-12, Sep. 2011.

[14] S. K. Mitra, R. Rajesh, R. K. Mishra, P. Rai, S. Vahikar, and P. Singhal, "Comparative evaluation of FNAC, core needle biopsy and excisional biopsy in subtyping of breast lesions," Trop. J. Pathol. Microbiol., vol. 2, no. 1, pp. 915, 2016.

[15] S. I. Panjvani et al., "Utility of Fine Needle Aspiration Cytology in the Evaluation of Breast Lesions," J. Clin. DIAGNOSTIC Res., vol. 7, no. 12, pp. 2777-2779, 2013.

[16] H. Panwar, P. Ingle, T. Santosh, V. Singh, A. Bugalia, and N. Hussain, "FNAC of Breast Lesions with Special Reference to IAC Standardized Reporting and Comparative Study of Cytohistological Grading of Breast Carcinoma.," J. Cytol., vol. 37, no. 1, pp. 34-39, 2008.

[17] S. B. Sankaye and S. D. Dongre, "Cytological study of palpable breast lumps presenting in an Indian rural setup.," Indian J. Med. Paediatr. Oncol., vol. 35, no. 2, pp. 159-64, Apr. 2014.

[18] P. Kujur, "Fine-Needle Aspiration Cytology of the Palpable Breast Lump of 106 Cases and Correlation with Histologic Diagnosis: A Prospective Analysis," Int. J. Sci. study, vol. 3, no. 9, pp. 111-115, 2015.

[19] R. Saadaat, J. Abdul-Ghafar, A. M. Haidary, S. Rahmani, and N. Atta, "Age distribution and types of breast lesions among Afghan women diagnosed by fine needle aspiration cytology (FNAC) at a tertiary care centre in Afghanistan: a descriptive cross-sectional study," BMJ Open, vol. 10, no. 9, p. e037513, Sep. 2020.

[20] F. Aker et al., "Accuracy of fine-needle aspiration cytology in the diagnosis of breast cancer a single-center retrospective study from 
Turkey with cytohistological correlation in 733 Ultrasonography and Fine Needle Aspiration cases," Diagn. Cytopathol., vol. 43, no. 12, pp. 978-986, Dec. 2015.

[21] J. Mišković, A. Zoric, H. R. Mišković, and V. Soljic, "Diagnostic value of Fine Needle Aspiration Cytology for Breast tumors," ACTA Clin. Croat., vol. 55, no. 4, pp. 625-28, 2016 Cytology in the Diagnosis of Malignant Breast Lesions," J. Clin. DIAGNOSTIC Res., vol. 7, no. 12 , pp. 2847-2850, 2013,

[23] O. N. Alema, A. M. Gakwaya, and D. Wamala, "Comparision of Fine Needle Aspiration Cytology and Fine Needle Sampling [22] Y. S. Takhellambam, S. S. Lourembam, O. without Aspiration in Diagnosis of Palpable S. Sapam, R. S. Kshetrimayum, B. S. Breast Lumps in Mulago Hospital.," East Cent. Ningthoujam, and T. Khan, "Comparison of African J. Surg., vol. 17, no. 1, pp. 104-11, 2012. 